\title{
How Research Technology and Innovation helps improve primary health care through tele medicine
}

\author{
Article by Surya Narayanan Sethumadavan \\ MBBS, Masters in Occupational Medicine, Fellowship in Emergency Medicine \\ Email:-drsuryas@yahoo.com
}

\begin{abstract}
This article will look into how Research, Technology and Innovation have improved primary health care around the world through telemedicine. Telemedicine and tele health have revolutionised medical care across the globe.

Objective: The paper is an original manuscript which will go into the entire gamut of primary health care and how innovation and technological improvements have revolutionised the concept of telemedicine and how patients across the world have immensely benefitted from this.

Design: This article will be a narrative on the research and technological advances of telemedicine over the years. This will refer to several studies and research works undertaken by several luminaries in this field. The references will be duly documented at the end of this article.

Procedure: The procedure to be followed will be a chronological sequencing of telemedicine as it evolved over the years and how innovation and technology have improved the patient care at the primary health care setting.

Results: A review of the scholarly articles and other studies in this field have pointed to one palpable truth - Research Technology and Innovation have revolutionised way telemedicine and primary healthcare have evolved over the years. With technology driven telemedicine, primary health care has become easy, quick and very affordable.

Conclusion: The bedrock for global development is indeed Research Technology and Innovation. As a doctor, my focus on telemedicine reveals how, technology has touched the lives of millions of people worldwide and how telemedicine has revolutionised this niche market of healthcare.
\end{abstract}

Keywords: Research, Technology, Innovation, telemedicine, primary healthcare

\section{Introduction}

Primary healthcare is a facet of health which touches the life of every person born in this world. Be it the most advanced cash rich country or a country struggling with healthcare facilities, every person reaches out to primary healthcare system at some point or the other in their lives. Right from the time we are born till death, primary healthcare service providers are constantly reaching out to us and enriching our lives. This article will look into how primary healthcare has evolved over the years and how primary healthcare providers have used innovation and technology to reach out to millions of patients.

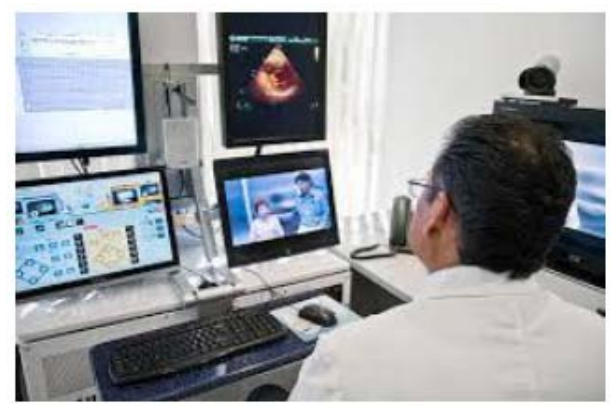


Over years the focus of primary healthcare has changed. And with this came unimaginable benefits. Nowadays the primary healthcare provider at your neighbourhood can do wonders in liaison with a super specialist sitting hundreds of miles away. This has been facilitated and improved by technological advances. The giant strides which technology has made over these years has made primary healthcare more efficient and patient friendly. The purpose of this article is to look into how innovation and technological advances have benefitted telemedicine at the primary healthcare setting.

\section{Methodology}

Several studies have been conducted over many years in different clinical settings in many geographically diverse environs spread out in different countries. These studies have been documented in this article and the references section at the end of this article lists these studies clearly.

A topic as vast as this which covers the entire realm of primary healthcare and how telemedicine has changed the game needs to be substantiated with scientific studies. Several medical luminaries have studied the efficacy of telemedicine. Initially the conservative world of doctors met telemedicine with some scepticism and doubt. But with time, the immense benefits of telemedicine and how doctors could visit the home of patients in remote areas started making people realise telemedicine was an easier and patient friendly option. In time, technology made things easier and more affordable and quicker.

\section{What is telemedicine?}

Telemedicine is the use of technology in the field of medicine in order to provide clinical health care at a distance. By doing this, crucial time delays and distance barriers are eliminated and healthcare is improved in remote areas. By using technology, medical information can be transmitted from remote primary healthcare location to a super specialist sitting in a sprawling super speciality tertiary healthcare centre in the city. A person can wonder what the big deal is. But by doing this, the patient sitting in a village gets to avail latest state of the art medical care which will normally not be available at a remote healthcare centre.

Telemedicine involves a growing field of medicine which involves using technology driven applications and services like video conferencing, emails, smart phones, iPad, wireless tools and several other tools using technological advances to connect a patient at a remote location with a doctor at an advanced care giving centre. An example would be a lady in a remote village in India suffering from skin rashes and itchy skin patches for years without realising what she's suffering from and what she needs to do to treat herself. Telemedicine can connect her with a dermatologist sitting several thousands of miles away in a super speciality hospital who can see the patient using an iPad and do a spot detection of her skin condition and initiate treatment for her.

Telemedicine started over 40 years ago with hospitals extending care to patients in remote areas. Over the years, the scope and reach of telemedicine or telehealth has exploded and now several hospitals have a dedicated department of telemedicine or telehealth dealing with home based healthcare. 


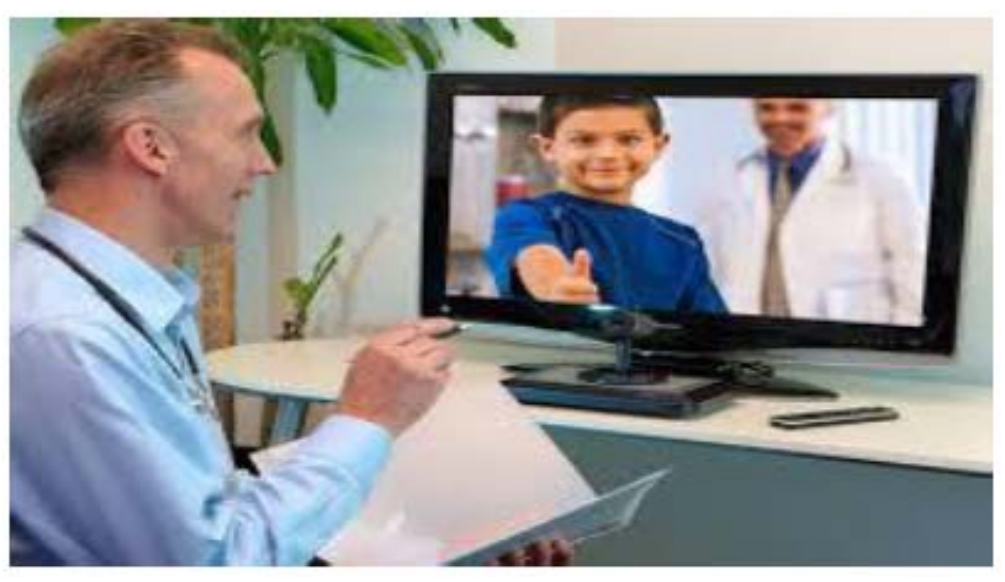

Technically speaking telemedicine is not a separate medical specialty. It is a mere extension of hospital facilities to a remote site. This could be tele cardiology, tele surgery, tele medicine, tele dermatology and so on. Telemedicine and telehealth are used interchangeably. Conducting patient consultations using video conferencing, using e Health Medical portals, escalating patient lab results to remote locations, continuing medical education to doctors sitting in different countries, transmission of images and videos of patients to doctors in other cities or countries to facilitate an expert opinion, remote monitoring of vital signs of a patient - including pulse, blood pressure, blood tests, BMI etc., call centres for patient health care and nursing care, home based health care providing nursing and doctor facilities, post discharge monitoring of patients at their homes - all this is part of telemedicine.

\section{What can telemedicine offer?}

Telemedicine can offer the following variations:

- Primary healthcare

- Specialist healthcare by referral to a specialist in a distant location

- Monitoring of patient in a remote location

- Dissemination and escalation of consumer medical information from one location to a specialist ( using safe and medically confidential fool proof methods)

- Medical education, seminars and conferences for medical practitioners - this e conference is an ideal example - being a doctor, this article is written here and would be sent to another country, thousands of miles away and would be read and assimilated by end users sitting in various locations. This is one such example - how technology and innovation has aided telemedicine.

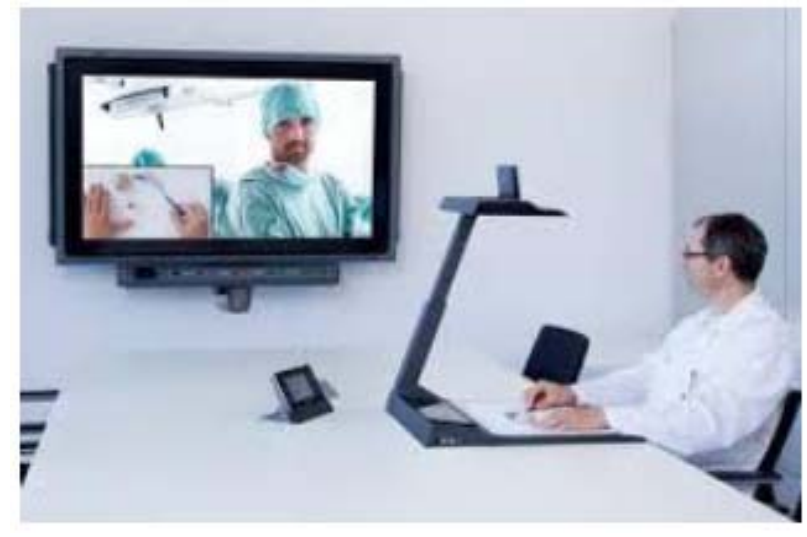

Now let us look at each of these in detail. 


\section{Primary healthcare}

A primary healthcare set up involves our friendly and affable neighbourhood General Physician (GP) and the patient. This GP model has been working for years on end and has helped thousands of people in every country in every town in every village. However, the GP model has a big disadvantage - critical cases, emergency patients and patients presenting with advanced disease conditions might place the GP in a position where the doctor might be unprepared to deal with the demands of the case. This is where telehealth steps in and unfolds its magic.

Using telemedicine, a specialist could refer the same patient to another super specialist without any travel involved. Without inconveniencing the patient, using technology, a referral could be done to another more qualified specialist who would be able to manage the case better.

With telemedicine, even a critical case or a case which needs expert evaluation can be assessed in a remote location by an expert sitting miles away and through technology driven tools, the GP can assist the patient and deliver a high level of medical care. Video conferencing can be used to connect a super specialist and the patient and this video can be saved for a later date during a follow up visit.

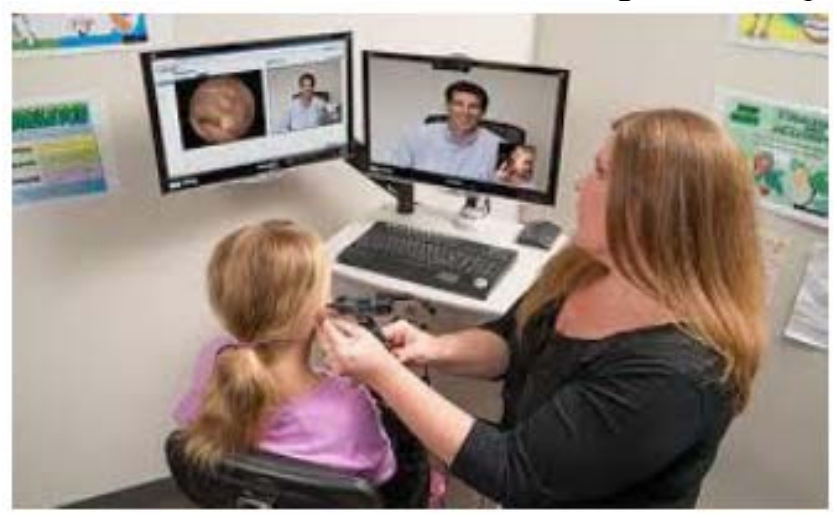

\section{Remote monitoring}

Highly advanced investigations like blood tests, cardiac scan or ECG, patient evaluation or monitoring of patient vitals can be done remote diagnostic testing. Nurses and doctors could be dispatched to a patient's home to provide home based healthcare at the comfy confines of a patient's home.

\section{Consumer medical information}

Using technology, patient's health information could be disseminated to other specialists to provide online consult. This would enable a fresh relook into the management of critical cases.

\section{Distance medical education}

Continuing medical education is an important facet for the development of any doctor or healthcare provider. Telemedicine is a crucial link in this journey. Latest breakthroughs in medical science and advances made in niche specialities are easily cascaded to the team of doctors across the globe. This helps doctors in equipping themselves better and in improving their services.

Though telemedicine brings in information technology and healthcare under one umbrella, the critical aspect here is to ensure the availability of technology for the end user. Without the availability of technology, remote healthcare will not be successful. The most common medium is interactive video.

Technology has grown consistently over the past 40 years but the main challenge is 
availability of niche cutting edge technological services for the end user. There is lack of consistency in availability of technology at the grassroots level. Though there are still several critics of telemedicine, it has been widely proved that telemedicine is indeed effective with good technological backup.

Telemedicine includes various specialities like tele radiology, tele pharmacy, tele dentistry, tele surgery, tele rehabilitative therapies, and tele public health. Costs have dramatically declined with advances in technology. With improvements in telecommunication, technology has advanced and this has resulted in declining costs over the past decade, there has been a steady growth in telemedicine. Telemedicine is not only of clinical benefit but cost effective too.

Wherever there is an element of distance between patient and doctor, it is called telemedicine. This is an umbrella term which includes primarily a patient doctor interaction that involves an element of distance. This is similar to using a radio by a ship captain to take advice from the shore or using technology by pilots to take advice from air traffic control.

Online health, e health, telemedicine and telehealth are all inter changeable names. Cost effectiveness and scientific evidence is very crucial for the success of telemedicine.

\section{Findings}

\section{Recent advances}

- A randomised control trial of home tele nursing was done which showed evidence of cost effectiveness.

- Outpatients are efficiently handled by electronic referrals

- In several locations, GP teleconsulting might turn out cheaper than traditional consulting.

- In several cases, where nurses were handling minor injuries, tele consultation with specialists turned out to be cost effective and quicker.

\section{Teleradiology}

A speciality of telemedicine where digital x-ray films, ultrasound scans, CT scans and MRI films are digitally transmitted from the primary healthcare centre to the radiologist sitting in a tertiary healthcare centre.

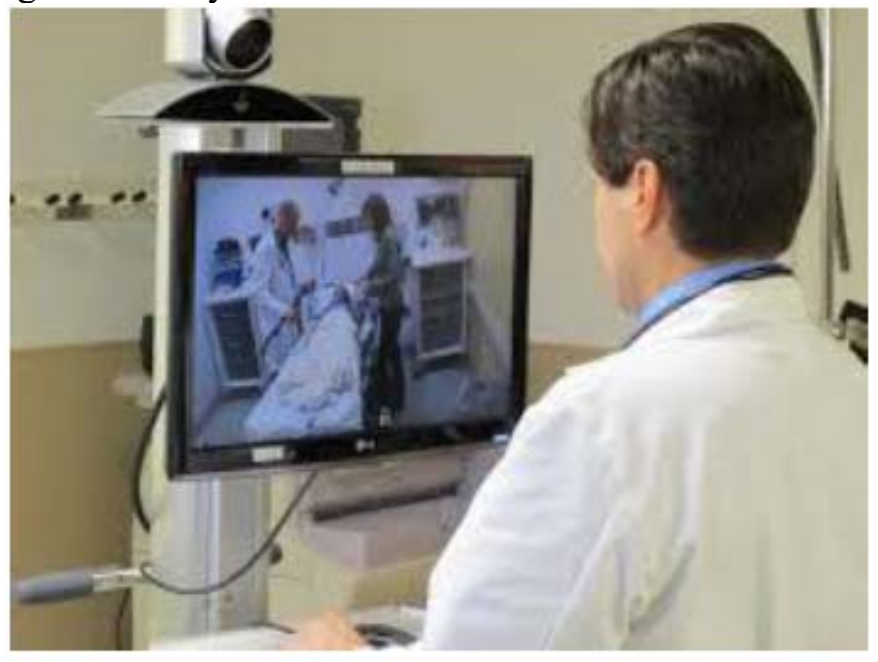

As is the case with any other form of telemedicine, some technology driven equipment are needed - a modern x-ray unit which can produce digital x-rays is needed. In certain set up, ultrasound scan machine, CT scan and MRI scan machines 
are also needed.

The receiving hospital also needs to have technologically compatible set up which can receive and escalate the radiology films to the specialist and there should be a method of returning the reports to the primary healthcare set up.

The advantages of having a tele radiology unit lies in the fact that there no need to maintain a radiologist or a radio diagnosis specialist in certain locations where the numbers of patients do not justify it. By using this tele radiology unit with technologically advanced support, patients living in remote locations can also avail specialist services.

The alternative would be to maintain a radiologist on location which might be an expensive proposition. On top of the money factor, getting specialist radiologists might not be feasible at all locations. Other options involve part time radiologists (visiting radio diagnosis specialist to visit patients at remote locations, once a week), the other option is to refer patients for radio diagnosis at a larger secondary or tertiary medical centre.

The cost effectiveness of tele radiology depends on the number of patients availing the services and costs involved in setting up the tele radiology unit and the revenue generated by the patients paying for the tele radiology services.

A Medline search of over 969 articles was conducted on telemedicine and this included cost effectiveness of tele medicine. Peer reviewed publications on telemedicine were studied. The keywords included "telemedicine", "telehealth", "online health" and "e health". The editorial board of Journal of Telemedicine and Telecare was also consulted.

Over the past several years, there has been a sudden spurt in the interest shown by the medical fraternity in implementing telemedicine for home based healthcare for chronically and terminally ill patients.

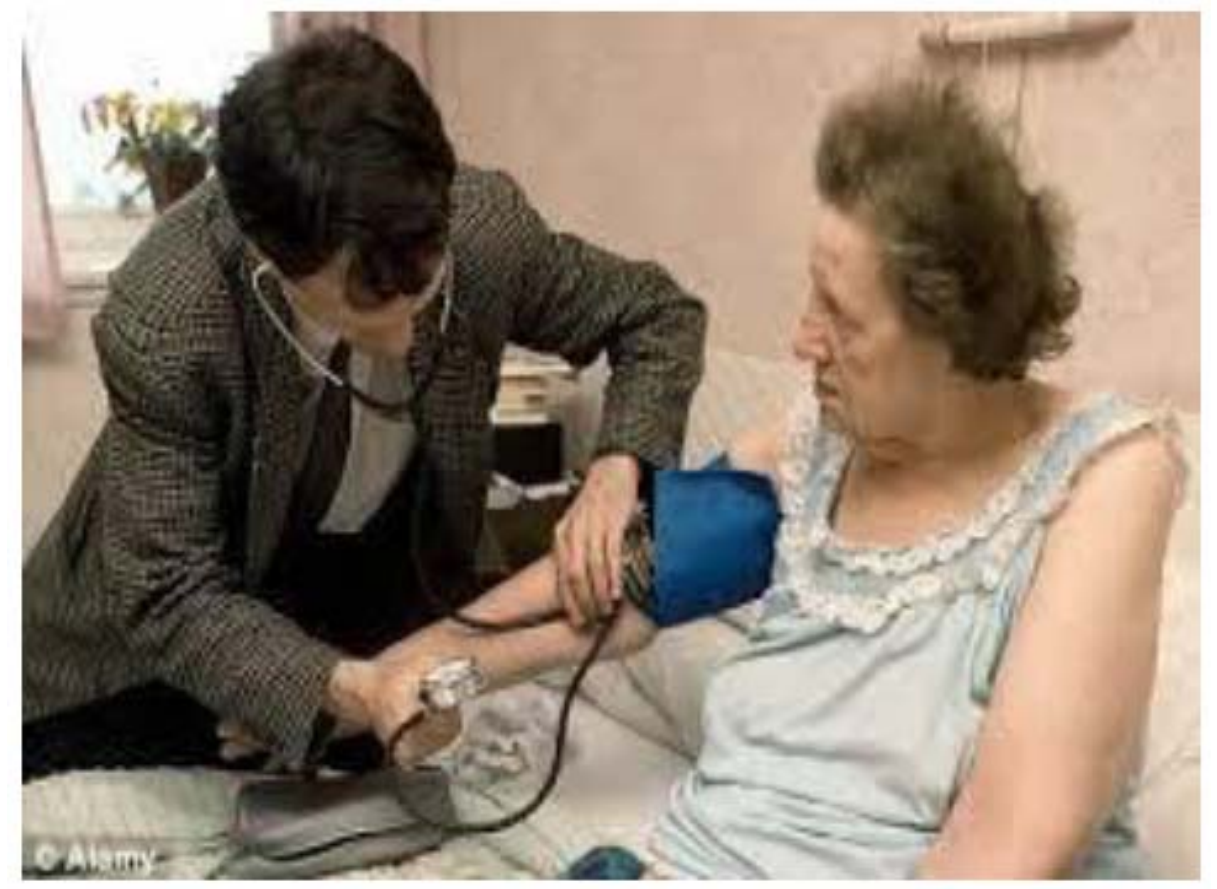

The advantages of this set up is that the patient is given care at the familiar home setting and patient compliance is very good. However, this involves a lot of expensive startup expenses involved in purchasing very expensive telecommunication equipment. This makes the concept quite expensive and almost comparable to hospital based expenses. 
One very significant study in home based healthcare using telemedicine and tele nursing was conducted by Kaiser Permanente. The first randomised controlled trial of home based nursing care for newly diagnosed patients with several diseases like cancer, diabetes, Chronic Obstructive Pulmonary Disease (COPD), Congestive Cardiac Failure (CCF), Anxiety and Cerebro Vascular Accident (CVA) were all selected and studied during this program. Patients were divided into two groups - the control group and the intervention group.

Digital stethoscope, Digital BP Apparatus and home videophones were given to the intervention group. Over 18 months the patients in both the groups were studied and patients in the telemedicine group received $17 \%$ less home visits by the nursing staff when compared to the control group.

Though the level of quality of care was the same in both groups, the satisfaction levels in telemedicine group was higher and they had less home visits and more telephonic contact with the care givers.

The best part was the average cost was $27 \%$ less than that of the care in the control group. (Outcomes of the Kaiser Permanente Tele-Home Health Research Project, Johnston B, Wheeler L, Deuser J, Sousa KH.) http://www.ncbi.nlm.nih.gov/pubmed/10664641

\section{Telenursing}

Telenursing is a very important link in the success of telehealth projects. One of the most critical aspects of telenursing is the availability of important tech devices for the nurses, which enable them to monitor the patients and simultaneously keep the data flowing to the specialist sitting miles away. In the Kaiser Permanente Trial, the equipment used were a digital BP apparatus, a videophone and an electronic stethoscope. The availability of these equipment might be a challenge at all locations.

To procure the equipment is a challenge and this is critical for the success of any telenursing project. Which is why it is easier to make such projects succeed at a community nursing home than in a private home because the cost of the equipment could pose a challenge to the public.

Some studies conducted in Hong Kong suggest that the trials of telemedicine maybe cost effective. (The role of telenursing in the provision of geriatric outreach services to residential homes in Hong Kong, Chan WM, Woo J, Hui E, Hjelm NM)

\section{Referrals to hospitals for specialist care using telemedicine}

A study conducted in Helsinki, Finland at the Peijas Hospital has shown a startling $52 \%$ referrals over a 20 month study period were much more cost effective than traditional out patient referrals.

This reduced expenses for the patient, decreased patient visits to hospitals and saved time, money and energy for the ailing patient. A teleconsultation through a video link was all that was needed by the hospital staff to facilitate this. Two groups of patients with similar ailments were studied and it was found that costs were seven times cheaper in the telemedicine group.

Similarly in Bangladesh, a study was conducted wherein a panel of specialists would give email support to the GPs and the general practitioners would then triage and treat the patients coming to them and the ease with which the patients were referred to higher medical centres was also determined by the tele email consultation. This scheme was cost effective and less time consuming.

\section{GP specialist nexus with telemedicine}

One of the biggest boons of telemedicine is the ease with which an inexperienced, new doctor sitting in a remote location is able to effortlessly and seamlessly consult with a specialist sitting miles away in a specialist tertiary medical centre. 
Traditionally, the GP sends the patient to a tertiary medical centre (usually a journey of several miles) and the handover is down through a handover form or a document. In telemedicine, the GP continues to manage the patient at the remote facility but the tertiary medical centre based Specialist is able to closely monitor the case through telemedicine. This avoids travel of the patient to a specialist hospital miles away. Three trials in New Zealand, Norway and UK have shown that teledermatology can be considered cost effective. The trials used real time video and they clearly demonstrated that significant travel time was reduced by telemedicine. This reduced a considerable burden for the patients. In rural areas of Scotland teledermatology is considered feasible, though the same might not be the case I n a sprawling city like London with many dermatologists available round the corner.

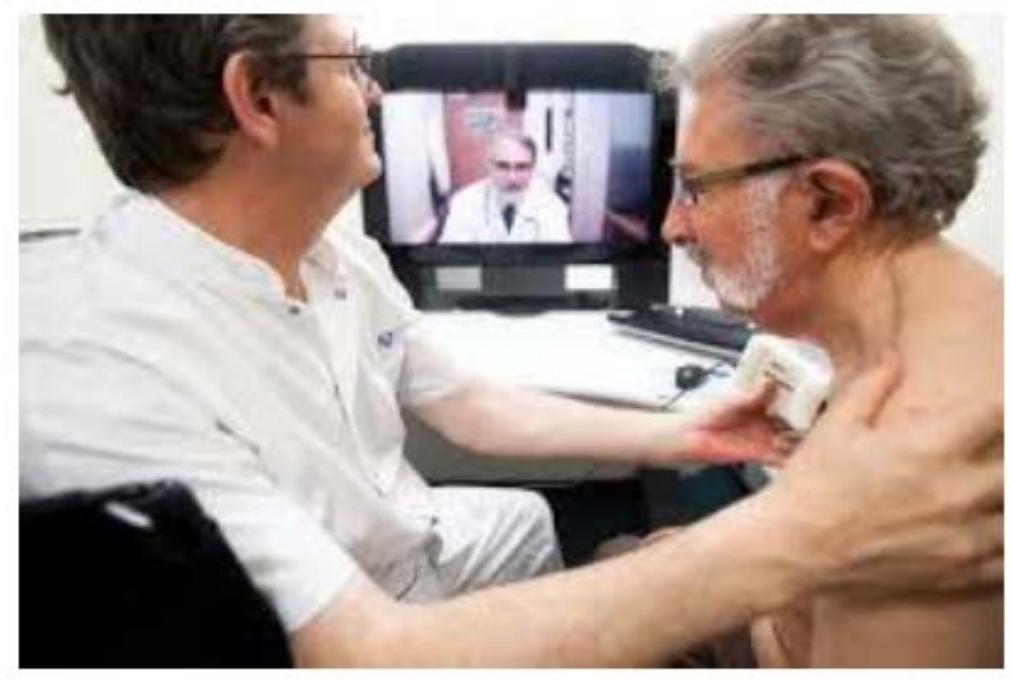

\section{Injury management and telemedicine}

All this said and done, one of the most promising and useful implications of telemedicine is to use real-time video by in the management of minor injuries. This has been studied in Scotland and in more than 20 injury units across UK. This has significantly demonstrated that nursing practitioners avoided unnecessary patient transfer and saved lots of money in managing minor injuries at their remote locations using real time video. A follow-up study by Central Middlesex Hospital has shown telemedicine is clinically safe and effective.

\section{Telephone call centres and telemedicine}

Many call centres which provide medical information and health information for public such as NHS Direct, try to triage callers into those who need emergency treatment, those who need primary healthcare referral and those who can safely treat themselves at home. This has shown to be effective and safe. Telephonic conversation usually is the first step of a triage process. Triage in medical parlance means - sorting out a patient based on the need for treatment. Based on the answers to a few questions, the callers are categorised into different groups. Based on this sorting or triaging, patients are managed accordingly.

\section{Online information sources}

\section{For health professionals}

http://tie.telemed.org Telemedicine Information Exchange database www.rsm.ac.uk/pub/jtt.htm Journal of Telemedicine and Telecare www.coh.uq.edu.au Centre for Online Health 
www.vh.org Virtual Hospital

www.rsm.ac.uk/pub/hii.htm Health Information on the Internet

\section{For patients}

www.healthcentre.org.uk UK health sites

Your Guide to E-Health by Peter Yellowlees. Published by University of Queensland Press as an e-book (www.uqp.uq.edu.au)

www.who.int/ith/english WHO travel advice (health)

\section{Conclusion}

\section{Future of telemedicine}

The future of conventional healthcare is intertwined with telemedicine. Access to healthcare is improved significantly in remote areas and there has been steep reduction in travel time and costs incurred by patients in remote locations. Geographical barriers are a major factor which help the proliferation of telemedicine, with several patients in lonely remote areas getting to meet a super specialist at the click of a computer mouse. Critics say telemedicine is too expensive and the equipment needed to start off telemedicine consultation are just little more than toys for the boys. Some others critique the equipment and telecommunications companies only provide a technical solution and do not understand the medical aspects. Another challenge in telemedicine is not just the availability of technology but the organisational challenge of how to take advantage of technology and how to train the usually conservative medical experts to become tech savvy and more technology driven.

Telemedicine has now entered the public realm and is now freely being accepted by many as a viable alternative. There are many who have saved a long trip to a referral hospital by employing telemedicine as a viable alternative and several studies have indeed shown in many settings, telemedicine is cost-effective. The long term challenge is using the latest cutting edge technology, sensitising the public and medical experts to the possibility of technology being a life saver and to walk a tight rope between reducing hospitalisation costs and the exorbitant costs of technological gadgets necessary for a telemedicine consult.

Technology and Innovation have revolutionised our lives in several ways and medical science is seeing a great sea change in which patients are treated and healed. Contrary to the conventional practice of a patient going to a doctor and waiting for several days and at times, several weeks to get a reasonable closure, telemedicine has saved time and money for the patient and it has brought the patient, the primary healthcare doctor and the specialist closer than ever before. With technological advances, patient satisfaction has increased. Telemedicine has evolved as a niche branch of Medicine and with newer research and technological breakthroughs, telemedicine is exploding and bringing smiles to the faces of patients and doctors. This article is an attempt to bring the latest advances and benefits of telemedicine to the discerning reader. It is hoped that the reader enjoys this as much as the author enjoyed writing this article. Let's hope with passing time, medical science evolves further and telemedicine becomes more affordable and easily accessible to the common man. With this, crucial time and money can be saved bringing in rich benefits for the patients.

\section{Conflict of interest}

Nothing to declare 
South American Journal of Medicine

Special Edition 2016

\section{References}

[1]. Armstrong IJ, Haston WS. Medical decision support for remote general practitioners using telemedicine. J Telemed Telecare. 1997;3:27-34.[PubMed]

[2]. Benger J. A review of minor injuries telemedicine. J Telemed Telecare 1999;5(suppl 3):S5-13

[3]. Bergmo TS. An economic analysis of teleradiology versus a visiting radiologist service. J Telemed Telecare. 1996;2:136-142.[PubMed]

[4]. Chan WM, Woo J, Hui H, Hjelm NM. The role of telenursing in the provision of geriatric outreach services to residential homes in Hong Kong. J Telemed Telecare. 2001;7:38-46.[PubMed]

[5]. Currell R, Urquhart C, Wainwright P, Lewis R. Telemedicine versus face to face patient care: effects on professional practice and health care outcomes. Cochrane Database Syst Rev 2000;(2):CD002098. [PubMed]

[6]. Harrison R, Clayton W, Wallace P. Can telemedicine be used to improve communication between primary and secondary care? BMJ. 1996;313:1377-1380. [PMC free article][PubMed]

[7]. Haukipuro K, Ohinmaa A, Winblad I, Linden T, Vuolio S. The feasibility of telemedicine for orthopaedic outpatient clinics — a randomized controlled trial. J Telemed Telecare. 2000;6:193198.[PubMed]

[8]. Hussain P, Melville D, Mannings R, Curry D, Kay D, Ford P. Evaluation of a training and diagnostic ultrasound service for general practitioners using narrowband ISDN. J Telemed Telecare. 1999;5(suppl 1):95-99.[PubMed]

[9]. Johnston B, Wheeler L, Deuser J, Sousa KH. Outcomes of the Kaiser Permanente Tele-Home Health Research Project. Arch Fam Med. 2000;9:40-45.[PubMed]

[10]. Keen J, Wyatt J. Back to basics on NHS networking. BMJ. 2000;321:875-878. [PMC free article][PubMed]

[11]. Larson A, Lynch DA, Zeligman B, Harlow C, Vanoni C, Thieme G, et al. Accuracy of diagnosis of subtle chest disease and subtle fractures with a teleradiology system. Am J Roentgenol. 1998;170:19-22.[PubMed]

[12]. Loane MA, Bloomer SE, Corbett R, Eedy DJ, Hicks N, Lotery HE, et al. A comparison of realtime and store-and-forward teledermatology: a cost-benefit study. Br J Dermatol. 2000;143:12411247.[PubMed]

[13]. Mair FS, Haycox A, May C, Williams T. A review of telemedicine cost-effectiveness studies. $J$ Telemed Telecare 2000;6(suppl 1):S38-40

[14]. McLaren P, Mohammedali A, Riley A, Gaughran F. Integrating interactive television-based psychiatric consultation into an urban community mental health service. J Telemed Telecare. 1999;5(suppl 1):100-102.[PubMed]

[15]. Minor injuries telemedicine. Proceedings of a meeting held in Belfast, 16 October 1998. J Telemed Telecare. 1999;5(suppl 3):1-50.

[16]. Munro J, Nicholl J, O'Cathain A, Knowles E. Impact of NHS Direct on demand for immediate care: observational study. BMJ. 2000;321:150-153. [PMC free article][PubMed]

[17]. Melzer SM, Poole SR. Computerized pediatric telephone triage and advice programs at children's hospitals: operating and financial characteristics. Arch Pediatr Adolesc Med. 1999;153:858863.[PubMed]

[18]. Oakley AM, Kerr P, Duffill M, Rademaker M, Fleischl P, Bradford N, et al. Patient cost-benefits of realtime teledermatology - a comparison of data from Northern Ireland and New Zealand. J Telemed Telecare. 2000;6:97-101.[PubMed]

[19]. Strode SW, Gustke S, Allen A. Technical and clinical progress in telemedicine. JAMA 1999;281:1066-8

[20]. Wootton R. Telemedicine: a cautious welcome. BMJ 1996;313:1375-7

[21]. Whitten P, Collins B. The diffusion of telemedicine. Sci Commun. 1997;19:21-40. 